\title{
Education as A Moderator in the Effect of Successful Aging on Mortality Risk in Different Genders of Elderly Chinese: a National Longitudinal Study (2011-2016)
}

\author{
Peiya Cao \\ West China School of Public Health and West China Fourth Hospital, Sichuan University. \\ Huiqiang Luo \\ West China School of Public Health and West China Fourth Hospital, Sichuan University. \\ Jijie Li \\ Department of Medical Records, West China Secondary University Hospital, Sichuan University. \\ Xiaohui Ren ( $\sim$ 422562728@qq.com ) \\ West China School of Public Health and West China Fourth Hospital, Sichuan University
}

\section{Research article}

Keywords: successful aging, education, mortality risk, elderly Chinese.

Posted Date: November 20th, 2020

DOI: https://doi.org/10.21203/rs.3.rs-107333/v1

License: (c) (i) This work is licensed under a Creative Commons Attribution 4.0 International License. Read Full License 


\section{Abstract}

Background Plenty of evidence has found that successful aging and its components were significantly associated with older adults' health, their achievement has a positive effect on reducing mortality rates. However, it is unclear whether education could modify the effect of successful aging on morality risk. Numerous literatures from worldwide were crosssectional and previous studies on the association between successful aging and mortality in China were quite few.

Methods Using four waves (2011-2012, 2013-2014, 2014-2015, 2015-2016) of a large nationally representative survey in China derived from CHARLS (China Health and Retirement Longitudinal Study) with 4,824 older adults aged 60 and older, this study aimed to evaluate the effect of successful aging and each of its components on mortality risk of different gender of older adults in China, we further discussed whether education was a moderator in this effect and investigated differences in results among males and females. Successful aging was measured by absence of major diseases, freedom from disability, high cognitive function, no depressive symptoms, and active social engagement in life. Cox proportional hazards models were applied to estimate the education's moderate effect on the relationship between successful aging and mortality after controlling a rich set of covariates that included demographics, socioeconomic status, and health behaviors.

Results We found that $15.18 \%(n=367)$ for males and $15.74 \%(n=379)$ for females were defined as successful aging and the mortality were $2.61 \%(n=63)$ for males and $3.45 \%(n=83)$ for females during the survey. The overall prevalence of successful aging in both genders were $12.5 \%(n=603)$ and the overall mortality rate was $3.03 \%(n=146)$.It is the first longitudinal study using national cohort data to research the educational effects on the association between mortality and successful aging, our study showed that the effect only existed in females aged 65-74 years old group with lower education.

Conclusions Education has the significant effect on the relationship between successful aging and mortality. Physical health is significantly associated with the achieving of successful aging among young older. More measures should be paid on improving mental health among the young female older with lower education to achieve successful aging and to against mortality and live longevity.

\section{Background}

Faced with life expectancy increased dramatically, the world's demographic structure has changed significantly[1]. It is predicted that almost 2 billion people will be 60 years or older by 2050 , accounting for $20 \%$ of the global population. China has the largest older adult population in the world, the population of older adults aged 60 years or older accounted for $16.1 \%$ of the total population in 2015 [2], and it will continue to keep this ranking until possibly as late as 2080 [3]. Aging is associated with cognitive functional problems[4], some major chronic diseases[5], and a decline of physical health[6], and other age-related problems, thereby posing challenges to health-care systems[7]. Social attention about aging has shifted from "how to live longer" to "how to age healthy"[8]. How can people age well? Many people, all around the world, regard good health as an important goal in their lives[9].

The concept of successful aging which is closely connected with good health and is regarded as a "gold standard" for evaluating older populations' health[10], has evolved for several decades. Successful aging was introduced by Robert J. Havighurst who first proposed the concept[11]. Rowe and Kahn discussed the operational concept of successful aging that encompasses three main criteria: low risk of disease and disability, maintenance of high physical and cognitive functioning, as well as active engagement in social and productive activities[12]. In recent years, the World Health Organization (WHO) reported the concept of active aging, which is "the process of optimizing opportunities for health, participation and security in order to enhance the quality life as people age"[13].

Although there are different defined successful aging across countries, cultures and literature, a majority of researches of successful aging has already paid attention to the factors which is associated with the achievement of successful aging 
and the prediction of future health outcomes result from successful aging[14]. A considerable number of studies have found that individual components (absence of major disease, freedom from disability, high cognitive function, no depressive symptoms, active social engagement in life) of successful aging were significantly associated with older adults' health[15], and their achievement has a positive effect on reducing mortality rates[16]. Older adults who were absent from major chronic diseases such as lung diseases significantly upper survival rate and life expectancy[17]. Disabled older people have a higher mortality rate than non-disabled counterparts[18]. High levels of depressive symptoms increased the risk of mortality[19]. Lastly, cognitive impairment (assessed by lower MMSE scores and slower reaction times) and physical dysfunction have been considered as critical factors resulting in lowered life expectancy[20, 21].

Education, as the common proxy for socioeconomic status (SES), makes it possible for individuals to master more knowledge about diseases, to understand the health treatments and cope with mechanisms for ill-health[22]. In empirical studies, numerous of them have provided evidence that education significantly contributed to the prediction of reaching successful aging[14, 23, 24]. Studies of 20th century found that higher educational level were linked with robust aging[2527]. Recent studies of Chinese elderly in Hong Kong and Shanghai have identified education as determinants of successful aging, higher educational level was related to higher rate of successful aging and the rate of successful aging was differ from years of education[28,29]. Education is also associated with psychosocial and biological conditions, two main criteria of successful aging, among the elderly, which means low levels of education were linked with poorer psychological function (depressive symptoms) and poorer biological conditions (diseases and disability)[30].

Higher socioeconomic status(age/gender/education), longer life expectancy and lower morbidity is positively associated with successful aging[31], indicating that better education is likely to prevent populations from high mortality risk, meaning the mortality rate might be higher who did not achieve successful aging than in their counterparts. Hence, it is of great interest to explore relationship between successful aging and mortality. However, there is little evidence to discuss health outcomes derived from successful aging and whether education can modify the effect of successful aging on morality risk. Furthermore, studies from worldwide were most cross-sectional and association between successful aging and mortality among Chinese elderly has remained unexplored thus far.

On the basis of above literature review, we hypothesized that successful aging would be associated with education and different levels of education, and education may modify the effect of successful aging on morality risk. Another primary hypothesis was that the effect as mentioned above experience gender difference, which means gender disparities may exist in the health indicators of mortality among successful aging population and non-successful aging population. So this prospective study first aims to evaluate the effect of successful aging and each of its components on mortality risk of the elderly aged 60 and over in China, the analyses of study population were classified into two group (60-74 years/75 years and above) because the effect may be different between young-old and old-old[26]; we further discussed whether education plays a moderator role in this effect using nationally representative longitudinal data.

\section{Methods}

\section{Study population}

CHARLS (China Health and Retirement Longitudinal Study) is a nationally representative follow-up survey, which is designed to investigate the economic and health of the populations aged 45 years old and above. The baseline national wave of CHARLS(W1) was being fielded in late summer 2011-March to 2012 and includes about 10,000 households in 150 counties/districts and 450 villages/resident committees. The individuals will be followed up every two years, Wave 2 (W2) was fielded from 2012 to 2013 and Wave 3 (W3) from 2015 to 2016. A special life history wave was fielded from 2014 to 2015 (W4) [32]. CHARLS questionnaires include information about self-reported and objective measures of health among middle-aged and elderly in China, these include health status and functioning, general health, physician-diagnosed chronic illnesses, lifestyle and health-related behaviors, subjective expectation of mortality, activities of daily living (ADL), cognition 
testing, depression and so on. The Biomedical Ethics Review Committee of Peking University (IRB00001052-11015) gave the ethic approval and allowed the CHARLS research group to collect data. Requiring all interviewees to sign informed consent was the first step of the study.

The study sample was derived from the baseline data W1 and follow-up data W2, W3 and W4 and specially was those who could be followed-up in the 4-year period, thus, as shown in Fig. 1, a total of 10,517 participants were investigated and 5,593 participants were excluded from the study for the following reasons: (1) samples with missing or invalid demographic data (e.g. education, marital status, smoking, drinking); (2) samples with two missing key relevant variables (e.g. successful aging and mortality information). The study population in the final analysis was 4,824, the demographic characteristics of the study population was in line with the original sample. The study population classified by gender and age were 1,954, 1,912, 463, 495 for males aged 60-74, females aged 60-74, males aged 75 and above, females aged 75 and above, respectively.

\section{Definition of successful aging}

Our concept of successful aging took the definition of Rowe and Kahn[12], including the following 5 components: 1) absence of major diseases, 2) freedom from disability, 3) high cognitive function, 4) no depressive symptoms, 5) active social engagement in life.

1 Absence of major diseases: To judge the status of chronic diseases, respondents were asked by the following question: "Have you been diagnosed with conditions listed below by a doctor?" The conditions include cancer, chronic lung disease, diabetes, heart disease and stroke. The research indicated that those diseases mentioned above may cause major disease burden for the elder[33], the respondents were classified as having no major diseases if they reported have no any of the above five chronic diseases.

2 Freedom from disability: The activities of daily living (ADL) scale was used to assess the ADLs[34], according to the following questions: "Because of a physical, mental, emotional or memory problem, do you have any difficulty with one type of everyday activity, excluding any that you expect to last less than three months?" The everyday activities include dressing, bathing, or showering, eating, getting into or out of bed, using the toilet, and controlling urination and defecation. Respondents were classified as having no disability if they reported that they had no difficulty with the everyday activities of six items mentioned above.

3 High cognitive function[35] : Cognitive function was assessed with the Telephone Interview of Cognitive Status (TICS). This includes both immediate and delayed recall of ten words on a list, serial subtraction of seven from 100 (up to five times), and naming the day of the week, month, day, year, and season, and drawing the picture. The score of cognitive function ranged from 0 to 21 . Respondents were considered to have high cognitive functioning if they achieved a median or above score, and the median score was 11.

4 No depressive symptoms: Depressive symptoms were assessed using the 10-item CES-D (Epidemiological Studies Depression Scale short form). The cut-off value is less than 10 points, which was used to identify no depressive symptoms.

5 Active social engagement in life: Respondents were defined as being actively social engaged if they participate in any of the following types of social groups: voluntary or charity work, provided help to family, friends, or neighbors, gone to a sport, social, or other kind of club in the month preceding the interview. The participant who met all five indicator criteria mentioned above was defined as "successful aging", otherwise as "non-successful aging".

\section{Other variables}

Control variables includes following factors: age (60-74 years/75 years or older), gender (Male/Female), education level (Primary school and below/Junior high school or above), income (low: <650 yuan, medium: 650 - 10,058 yuan,high: $>10,058$ yuan) marital status (Married/Cohabitating/Divorced/Separated/Widowed/Never married), community type 
(Rural/Urban), smoking (Yes/No/Quit), and drinking (Drink more than once a month/Drink but less than once a month/Do not drink).

\section{Mortality}

Participants enrolled in W1 were followed up in W2, W3 and W4. We collected the all-cause mortality and survival information of the respondents during the four wave surveys (2011-2016). W2 recorded of the respondents' both status information (dead or alive) and death time, while W3 and W4 only provided the interview status information (dead or alive). We recorded and calculated the survival time of those who had the accurate all-cause death time by the interval between the interview time of W1 and the death time in W2. If respondents' accurate death time was not available, we calculated the specific value by the interval between the interview time of W1 and the specific wave with death information and then defined the median of this value as the survival time. The survival time of those who were alive during whole follow-up interview was the interval between W1 and W4.

\section{Statistics analysis}

All descriptive statistical analyses were performed by gender, frequencies and percentages were calculated to describe the sample. We first used chi-square test to compare individual characteristics (including dichotomous or categorical variables) with and without successful aging. Next, the survival analysis was used to examine the association between successful aging and all-cause mortality, the Cox proportional hazards regression models were used to estimate the unadjusted and adjusted hazard rations (HRs) and 95\% confidence intervals (CLs) of successful aging. At last, whether the education could modify the effect of successful aging on the all-cause mortality was assessed, in this stage, we also used the Cox proportional hazards regression models to examine the educational mediating effect on all-cause mortality among successful aging and non-successful aging populations. All statistical analyses were performed by SAS 9.3. The significance level was set at 0.05 .

\section{Results}

Table 1 shows the respondents' baseline characteristics according to successful aging. In the group aged 60-74 years old, $15.20 \%(n=297)$ for males and $15.95 \%(n=305)$ for females were defined as successful aging according to our definition, the overall prevalence of successful aging in both genders were12.5\% $(n=603)$. Among males and females, people with high education level were likely to achieve successful aging compared with lower education $(P<0.001)$. Higher income makes it possible for people to achieve successful aging $(P<0.01)$. People keeping the drinking habits (Drink but less than once a month and Drink more than once a month, $\mathrm{P}<0.001$ ) were more likely to achieve aging successfully, compared with the people (Do not drink). In addition, smokers were more likely to be successfully aging in male group $(P=0.001)$. For the

group of 75 years old and above, the successful aging rate was $15.12 \%(n=70)$ for males and $14.95 \%(n=74)$ for females respectively. The connection between successful aging and education was similar among the group aged 60-74 years old. Other details of characteristics of participants were shown in Table 1. 
Table 1

Characteristics of Study Population According to Baseline Successful Aging by Gender

\begin{tabular}{|c|c|c|c|c|c|c|}
\hline \multirow[t]{2}{*}{ Characteristics } & \multicolumn{2}{|l|}{ Male (N (\%)) } & \multirow[t]{2}{*}{$P$} & \multicolumn{2}{|c|}{ Female (N (\%)) } & \multirow[t]{2}{*}{$P$} \\
\hline & $\begin{array}{l}\text { successful } \\
\text { aging }\end{array}$ & $\begin{array}{l}\text { Non- } \\
\text { successful } \\
\text { aging }\end{array}$ & & $\begin{array}{l}\text { successful } \\
\text { aging }\end{array}$ & $\begin{array}{l}\text { Non- } \\
\text { successful } \\
\text { aging }\end{array}$ & \\
\hline $60-74$ years old & $297(15.20)$ & 1657(84.80) & & $305(15.95)$ & $1607(84.05)$ & \\
\hline \multicolumn{7}{|l|}{ Education level } \\
\hline Primary school and below & 133(10.33) & 1154(89.67) & \multirow{2}{*}{$<.001$} & $126(9.91)$ & 1146(90.09) & \multirow{2}{*}{$<.001$} \\
\hline Junior high school or above & 164(24.59) & $503(75.41)$ & & $179(27.97)$ & $461(72.03)$ & \\
\hline \multicolumn{7}{|l|}{ Marital status } \\
\hline Married/Cohabitating & $260(14.91)$ & 1484(85.09) & \multirow[t]{2}{*}{0.301} & $241(16.07)$ & $1259(83.93)$ & \multirow[t]{2}{*}{0.794} \\
\hline $\begin{array}{l}\text { Divorced/Separated/Widowed/Never } \\
\text { married }\end{array}$ & $37(17.62)$ & 173(82.38) & & $64(15.53)$ & $348(84.47)$ & \\
\hline \multicolumn{7}{|l|}{ Community type } \\
\hline Rural & 232(15.95) & 1223(84.05) & \multirow[t]{2}{*}{0.117} & $245(16.04)$ & 1282(83.96) & \multirow[t]{2}{*}{0.826} \\
\hline Urban & 65(13.03) & 434(86.97) & & $60(15.58)$ & $325(84.42)$ & \\
\hline \multicolumn{7}{|l|}{ Income } \\
\hline$<650$ & $57(11.75)$ & $428(88.25)$ & $<.001$ & $64(13.22)$ & $420(86.78)$ & 0.002 \\
\hline $650-10058$ & 137(13.39) & $886(86.61)$ & & $132(14.63)$ & $770(85.37)$ & \\
\hline$>10058$ & 103(23.09) & $343(76.91)$ & & 109(20.72) & $417(79.28)$ & \\
\hline \multicolumn{7}{|l|}{ Smoking } \\
\hline Yes & $121(18.67)$ & $527(81.33)$ & \multirow[t]{3}{*}{0.001} & $106(18.50)$ & $467(81.50)$ & \multirow[t]{3}{*}{0.111} \\
\hline No & $142(12.61)$ & 984(87.39) & & $170(14.60)$ & $994(85.40)$ & \\
\hline Quit & $34(18.89)$ & 146(81.11) & & $29(16.57)$ & 146(83.43) & \\
\hline \multicolumn{7}{|l|}{ Drinking } \\
\hline Drink more than once a month & $29(19.46)$ & $120(80.54)$ & \multirow{3}{*}{$<.001$} & 28(18.92) & $120(81.08)$ & \multirow{4}{*}{$<.001$} \\
\hline Drink but less than once a month & $105(21.60)$ & $381(78.40)$ & & $98(21.35)$ & $361(78.65)$ & \\
\hline Do not drink & $163(12.36)$ & 1156(87.64) & & 179(13.72) & $1126(86.28)$ & \\
\hline 75 years old and above & $70(15.12)$ & $393(84.88)$ & & $74(14.95)$ & $421(85.05)$ & \\
\hline \multicolumn{7}{|l|}{ Education level } \\
\hline Primary school and below & $30(10.00)$ & $270(90.00)$ & \multirow{2}{*}{$<.001$} & $34(10.30)$ & 296(89.70) & \multirow{2}{*}{$\dot{0} 001$} \\
\hline Junior high school or above & $40(24.54)$ & $123(75.46)$ & & $40(24.24)$ & $125(75.76)$ & \\
\hline \multicolumn{7}{|l|}{ Marital status } \\
\hline Married/Cohabitating & $50(14.88)$ & $286(85.12)$ & 0.816 & $25(13.97)$ & 154(86.03) & 0.644 \\
\hline
\end{tabular}




\begin{tabular}{|c|c|c|c|c|c|c|}
\hline \multirow[t]{2}{*}{ Characteristics } & \multicolumn{2}{|l|}{ Male (N (\%)) } & \multirow[t]{2}{*}{$P$} & \multicolumn{2}{|c|}{ Female (N (\%)) } & \multirow[t]{2}{*}{$P$} \\
\hline & $\begin{array}{l}\text { successful } \\
\text { aging }\end{array}$ & $\begin{array}{l}\text { Non- } \\
\text { successful } \\
\text { aging }\end{array}$ & & $\begin{array}{l}\text { successful } \\
\text { aging }\end{array}$ & $\begin{array}{l}\text { Non- } \\
\text { successful } \\
\text { aging }\end{array}$ & \\
\hline $\begin{array}{l}\text { Divorced/Separated/Widowed/Never } \\
\text { married }\end{array}$ & $20(15.75)$ & 107(84.25) & & 49(15.51) & $267(84.49)$ & \\
\hline \multicolumn{7}{|l|}{ Community type } \\
\hline Rural & $46(13.90)$ & 285(86.10) & \multirow[t]{2}{*}{0.245} & $54(14.29)$ & $324(85.71)$ & \multirow[t]{2}{*}{0.457} \\
\hline Urban & 24(18.18) & 108(81.82) & & 20(17.09) & $97(82.91)$ & \\
\hline \multicolumn{7}{|l|}{ Income } \\
\hline$<650$ & $12(10.91)$ & 98(89.09) & \multirow[t]{2}{*}{0.051} & $16(12.90)$ & $108(87.10)$ & \multirow[t]{2}{*}{0.458} \\
\hline $650-10,058$ & $33(13.81)$ & 206(86.19) & & $36(14.34)$ & 215(85.66) & \\
\hline$>10,058$ & 25(21.93) & $89(78.07)$ & & 22(18.33) & $98(81.67)$ & \\
\hline \multicolumn{7}{|l|}{ Smoking } \\
\hline Yes & $22(15.07)$ & 12484.93) & \multirow[t]{3}{*}{0.579} & $26(16.35)$ & 133(83.65) & \multirow[t]{3}{*}{0.694} \\
\hline No & $38(14.23)$ & 229(85.77) & & $41(13.85)$ & 255(86.15) & \\
\hline Quit & $10(20.00)$ & $40(80.00)$ & & $7(17.50)$ & $33(82.50)$ & \\
\hline \multicolumn{7}{|l|}{ Drinking } \\
\hline Drink more than once a month & $8(22.86)$ & 27(77.14) & \multirow[t]{3}{*}{0.325} & $9(18.00)$ & $41(82.00)$ & \multirow[t]{3}{*}{0.141} \\
\hline Drink but less than once a month & 20(16.39) & 102(83.61) & & $27(19.29)$ & 113(80.71) & \\
\hline Do not drink & 42(13.73) & 264(86.27) & & $38(12.46)$ & $267(87.54)$ & \\
\hline
\end{tabular}

As Table 2 shows, among population aged $60-74$, the mortality rate of females (3.50\%) was higher than that of males $(2.46 \%)$, although the difference was not significant $(P=0.055)$. Moreover, the distribution of the association between successful aging and its components and mortality differed by gender, the association between successful aging and mortality was significant in females $(P=0.033)$, but not in males $(P=0.200)$. Specifically speaking, individual components comprising successful aging except "Active social engagement in life $(P=0.642)$ " in females were associated with mortality significantly, and those significant correlation in males only existed in " Absence of major disease $(P<0.001)$ " and" Freedom from disability $(\mathrm{P}<0.001)$ ". Among group aged 75 and above, the mortality rate of males and females were similar (males = $3.24 \%$, female $=3.23 \%, P=0.995)$. In addition, both males and females showed the same correlation trend between successful aging and its components and mortality except " Absence of major disease $(P=0.004)$ " was associated with mortality in males and "High cognitive function $(P=0.021)$ " was associated with mortality in females. 
Table 2

Association between Successful Aging and Mortality by Gender Based on Baseline Successful Aging

\begin{tabular}{|c|c|c|c|c|c|c|}
\hline & \multicolumn{2}{|l|}{ Male (N (\%)) } & \multirow[t]{2}{*}{$P$} & \multicolumn{2}{|l|}{ Female (N (\%)) } & \multirow[t]{2}{*}{$P$} \\
\hline & $\begin{array}{l}\text { Mortality } \\
\text { incidence cases }\end{array}$ & $\begin{array}{l}\text { Follow-up } \\
\text { cases }\end{array}$ & & $\begin{array}{l}\text { Mortality } \\
\text { incidence cases }\end{array}$ & $\begin{array}{l}\text { Follow-up } \\
\text { cases }\end{array}$ & \\
\hline $60-74$ years old & $48(2.46)$ & 1954 & & $67(3.50)$ & 1912 & \\
\hline \multicolumn{7}{|l|}{ Successful aging } \\
\hline Yes & $4(1.35)$ & 297 & \multirow[t]{2}{*}{0.200} & $4(1.31)$ & 305 & \multirow[t]{2}{*}{0.033} \\
\hline No & $44(2.66)$ & 1657 & & $63(3.92)$ & 1607 & \\
\hline \multicolumn{7}{|l|}{$\begin{array}{l}\text { Absence of major } \\
\text { diseases }\end{array}$} \\
\hline Yes & $24(1.62)$ & 1480 & \multirow{2}{*}{$\dot{0} 0.001$} & $39(2.76)$ & 1411 & \multirow[t]{2}{*}{0.003} \\
\hline No & $24(5.06)$ & 474 & & $28(5.59)$ & 501 & \\
\hline \multicolumn{7}{|l|}{$\begin{array}{l}\text { Freedom from } \\
\text { disability }\end{array}$} \\
\hline Yes & $31(1.83)$ & 1693 & \multirow{2}{*}{$<.001$} & $50(3.05)$ & 1642 & \multirow[t]{2}{*}{0.009} \\
\hline No & $17(6.51)$ & 261 & & $17(6.30)$ & 270 & \\
\hline \multicolumn{7}{|l|}{ High cognitive function } \\
\hline Yes & $23(2.15)$ & 1069 & \multirow[t]{2}{*}{0.356} & $23(2.17)$ & 1058 & \multirow[t]{2}{*}{0.001} \\
\hline No & $25(2.82)$ & 885 & & $44(5.15)$ & 854 & \\
\hline \multicolumn{7}{|l|}{$\begin{array}{l}\text { No depressive } \\
\text { symptoms }\end{array}$} \\
\hline Yes & $29(2.35)$ & 1236 & \multirow[t]{2}{*}{0.676} & $34(2.77)$ & 1227 & \multirow[t]{2}{*}{0.023} \\
\hline No & $19(2.65)$ & 718 & & $33(4.82)$ & 685 & \\
\hline \multicolumn{7}{|l|}{$\begin{array}{l}\text { Active social } \\
\text { engagement in life }\end{array}$} \\
\hline Yes & $19(1.90)$ & 1001 & \multirow[t]{2}{*}{0.112} & $32(3.30)$ & 969 & \multirow[t]{2}{*}{0.642} \\
\hline No & $29(3.04)$ & 953 & & $35(3.71)$ & 943 & \\
\hline 75 years old and above & $15(3.24)$ & 463 & & 16(3.23) & 495 & \\
\hline \multicolumn{7}{|l|}{ Successful aging } \\
\hline Yes & $1(1.43)$ & 70 & \multirow[t]{2}{*}{0.359} & $0(0.00)$ & 74 & \multirow[t]{2}{*}{0.287} \\
\hline No & $14(3.56)$ & 393 & & $16(3.80)$ & 421 & \\
\hline \multicolumn{7}{|l|}{$\begin{array}{l}\text { Absence of major } \\
\text { diseases }\end{array}$} \\
\hline Yes & 7(1.93) & 363 & \multirow[t]{2}{*}{0.004} & 11(2.98) & 369 & \multirow[t]{2}{*}{0.554} \\
\hline No & $8(8.00)$ & 100 & & $5(3.97)$ & 126 & \\
\hline $\begin{array}{l}\text { Freedom from } \\
\text { disability }\end{array}$ & & & & & & \\
\hline
\end{tabular}




\begin{tabular}{|c|c|c|c|c|c|c|}
\hline & \multicolumn{2}{|l|}{ Male (N (\%)) } & \multirow[t]{2}{*}{$P$} & \multicolumn{2}{|l|}{ Female (N (\%)) } & \multirow[t]{2}{*}{$P$} \\
\hline & $\begin{array}{l}\text { Mortality } \\
\text { incidence cases }\end{array}$ & $\begin{array}{l}\text { Follow-up } \\
\text { cases }\end{array}$ & & $\begin{array}{l}\text { Mortality } \\
\text { incidence cases }\end{array}$ & $\begin{array}{l}\text { Follow-up } \\
\text { cases }\end{array}$ & \\
\hline Yes & $11(2.84)$ & 387 & \multirow[t]{2}{*}{0.297} & 14(3.33) & 421 & \multirow[t]{2}{*}{0.791} \\
\hline No & $4(5.26)$ & 76 & & $2(2.70)$ & 74 & \\
\hline \multicolumn{7}{|c|}{ High cognitive function } \\
\hline Yes & $5(2.00)$ & 250 & \multirow[t]{2}{*}{0.120} & $4(1.44)$ & 278 & \multirow[t]{2}{*}{0.021} \\
\hline No & $10(4.69)$ & 213 & & $12(5.53)$ & 217 & \\
\hline \multicolumn{7}{|c|}{$\begin{array}{l}\text { No depressive } \\
\text { symptoms }\end{array}$} \\
\hline Yes & $9(3.10)$ & 290 & \multirow[t]{2}{*}{0.865} & $11(3.68)$ & 299 & \multirow[t]{2}{*}{0.503} \\
\hline No & $6(3.47)$ & 173 & & $5(2.55)$ & 196 & \\
\hline \multicolumn{7}{|c|}{$\begin{array}{l}\text { Active social } \\
\text { engagement in life }\end{array}$} \\
\hline Yes & $7(3.04)$ & 230 & \multirow[t]{2}{*}{0.815} & $4(1.44)$ & 278 & \multirow[t]{2}{*}{0.362} \\
\hline No & $8(3.43)$ & 233 & & $12(5.53)$ & 217 & \\
\hline
\end{tabular}

In order to assess the risk of mortality onset, the Cox proportional hazards models were used to show relevant results during the follow-up survey according to the successful aging at baseline participants (Table 3).In total, after adjusting education, health behavioral and relevant influencing factors, the association between successful aging and mortality was only observed in the group aged $60-74$ in females $(H R=3.105,95 \% \mathrm{Cl}=1.128-8.543)$. However, there was no association between successful aging and mortality significantly in males. In addition, owing to the restriction of data, the females aged 75 and above cannot observe similar correlation (females defined as aging successfully had no death data at that age stage).

Table $3 \mathrm{HR}(95 \% \mathrm{Cl})$ of All-Cause Mortality of Successful Aging and Its Component 


\begin{tabular}{|c|c|c|c|c|}
\hline & \multicolumn{2}{|c|}{ Male (HR 95\%Cl) } & \multicolumn{2}{|c|}{ Female (HR 95\%Cl) } \\
\hline & Unadjusted & Adjusted & Unadjusted & Adjusted \\
\hline \multicolumn{5}{|l|}{$60-74$ years old } \\
\hline Successful aging & Ref. & & Ref. & \\
\hline Non-successful aging & $\begin{array}{l}1.953(0.702- \\
5.436)\end{array}$ & $\begin{array}{l}2.068(0.739- \\
5.784)\end{array}$ & $\begin{array}{l}2.994(1.090- \\
8.226)^{\star}\end{array}$ & $\begin{array}{l}3.105(1.128- \\
8.543)^{\star}\end{array}$ \\
\hline Absence of major diseases & Ref. & & Ref. & \\
\hline Major disease & $\begin{array}{l}3.195(1.815- \\
5.626)^{\star \star \star}\end{array}$ & $\begin{array}{l}3.299(1.865- \\
5.835)^{\star \star \star}\end{array}$ & $\begin{array}{l}2.063(1.269- \\
3.352)^{\star \star}\end{array}$ & $\begin{array}{l}2.080(1.276- \\
3.380)^{\star \star}\end{array}$ \\
\hline Freedom from disability & Ref. & & Ref. & \\
\hline Disability & $\begin{array}{l}3.670(2.031- \\
6.631)^{\star \star \star}\end{array}$ & $\begin{array}{l}3.868(2.132- \\
7.018)^{\star \star \star}\end{array}$ & $\begin{array}{l}2.085(1.202- \\
3.614)^{\star \star}\end{array}$ & $\begin{array}{l}2.169(1.246- \\
3.777)^{\star \star}\end{array}$ \\
\hline High cognitive function & Ref. & & Ref. & \\
\hline Not high cognitive function & $\begin{array}{l}1.306(0.741- \\
2.300)\end{array}$ & $\begin{array}{l}1.374(0.775- \\
2.437)\end{array}$ & $\begin{array}{l}2.378(1.436- \\
3.938)^{\star \star}\end{array}$ & $\begin{array}{l}2.532(1.520- \\
4.217)^{\star \star \star}\end{array}$ \\
\hline No depressive symptoms & Ref. & & Ref. & \\
\hline Depressive symptoms & $\begin{array}{l}1.131(0.634- \\
2.017)\end{array}$ & $\begin{array}{l}1.164(0.651- \\
2.081)\end{array}$ & $\begin{array}{l}1.743(1.080- \\
2.814)^{\star}\end{array}$ & $\begin{array}{l}1.785(1.102- \\
2.890)^{\star}\end{array}$ \\
\hline $\begin{array}{l}\text { Active social engagement in } \\
\text { life }\end{array}$ & Ref. & & Ref. & \\
\hline $\begin{array}{l}\text { Not active social engagement } \\
\text { in life }\end{array}$ & $\begin{array}{l}1.598(0.896- \\
2.850)\end{array}$ & $\begin{array}{l}1.633(0.914- \\
2.918)\end{array}$ & $\begin{array}{l}1.121(0.694- \\
1.810)\end{array}$ & $\begin{array}{l}1.140(0.705- \\
1.841)\end{array}$ \\
\hline \multicolumn{5}{|l|}{75 years old and above } \\
\hline Successful aging & Ref. & & Ref. & \\
\hline Non-successful aging & $\begin{array}{l}2.583(0.340- \\
19.642)\end{array}$ & $\begin{array}{l}2.677(0.346- \\
20.702)\end{array}$ & NA & \\
\hline Absence of major diseases & Ref. & & Ref. & \\
\hline Major disease & $\begin{array}{l}4.367(1.583- \\
12.044)^{\star \star}\end{array}$ & $\begin{array}{l}4.027(1.435- \\
11.297)^{\star \star}\end{array}$ & $\begin{array}{l}1.375(0.478- \\
3.959)\end{array}$ & $\begin{array}{l}1.236(0.425- \\
3.598)\end{array}$ \\
\hline Freedom from disability & Ref. & & Ref. & \\
\hline Disability & $\begin{array}{l}1.839(0.586- \\
5.776)\end{array}$ & $\begin{array}{l}1.984(0.620- \\
6.352)\end{array}$ & $\begin{array}{l}0.819(0.186- \\
3.601)\end{array}$ & $\begin{array}{l}0.690(0.155- \\
3.081)\end{array}$ \\
\hline High cognitive function & Ref. & & Ref. & \\
\hline Not high cognitive function & $\begin{array}{l}2.341(0.800- \\
6.849)\end{array}$ & $\begin{array}{l}2.359(0.783- \\
7.110)\end{array}$ & $\begin{array}{l}3.802(1.226- \\
11.787)^{\star}\end{array}$ & $\begin{array}{l}3.008(0.958- \\
9.447)\end{array}$ \\
\hline No depressive symptoms & Ref. & & Ref. & \\
\hline Depressive symptoms & $\begin{array}{l}1.093(0.389- \\
3.072)\end{array}$ & $\begin{array}{l}1.293(0.441- \\
3.789)\end{array}$ & $\begin{array}{l}0.697(0.242- \\
2.006)\end{array}$ & $\begin{array}{l}0.661(0.229- \\
1.911)\end{array}$ \\
\hline $\begin{array}{l}\text { Active social engagement in } \\
\text { life }\end{array}$ & Ref. & & Ref. & \\
\hline Not active social engagement & $1.128(0.409-$ & 1.107(0.393- & $0.625(0.227-$ & $0.580(0.209-$ \\
\hline
\end{tabular}


${ }^{*} p<0.05^{* \star} p<0.01^{* \star *} p<0.001$.

a Unadjusted model.

${ }^{\text {b }}$ Adjusted for model 1 criteria and Marital status, Community type, Education, Income, Smoking, Drinking.

The study further explored if the education was a moderator in this effect of successful aging on morality risk (Table 4). Owing to there was no significant association between successful aging and mortality in the group of 75 years old and above, relevant analysis mainly on the participants aged 60-74 years old. Table 4 shows the significant correlation between successful aging and mortality only exist in females' group with the education level of primary school and below $(\mathrm{HR}=3.272,95 \% \mathrm{Cl}=1.019-10.507)$.

\section{Table 4 HRs of successful aging in 60-74 years old groups with different education level}

\begin{tabular}{|c|c|c|c|}
\hline & \multirow[t]{2}{*}{ successful aging } & \multicolumn{2}{|l|}{ Non-Successful aging } \\
\hline & & Male & Female \\
\hline \multicolumn{4}{|c|}{ Primary school and below (60-74 years old) } \\
\hline Unadjusted $^{a}$ & Ref. & $1.632(0.496-5.367)$ & $3.141(1.009-10.068) *$ \\
\hline Adjusted ${ }^{b}$ & Ref. & $1.727(0.522-5.716)$ & $3.272(1.019-10.507) *$ \\
\hline \multicolumn{4}{|c|}{ Junior high school or above (60-74 years old) } \\
\hline Unadjusted $^{\mathrm{a}}$ & Ref. & $2.925(0.388-22.053)$ & $2.623(0.343-20.056)$ \\
\hline Adjusted $^{b}$ & Ref. & $3.422(0.448-26.114)$ & $2.884(0.367-22.665)$ \\
\hline \multicolumn{4}{|l|}{ Note } \\
\hline \multicolumn{4}{|l|}{${ }^{*} p<0.05$} \\
\hline \multicolumn{4}{|c|}{ a Unadjusted model. } \\
\hline
\end{tabular}

\section{Discussion}

The study investigated the association between successful aging and mortality among different genders, and further explored education's role of moderating the effect of successful aging on mortality. We found the significant correlation between successful aging and its 5 components with mortality, meanwhile, those correlation existed gender differences. In addition, those significant association only existed in the group of females (60-74 years old) with education level of primary school and below.

The prevalence of successful aging among males and females were $15.20 \%$ and $15.95 \%$ respectively, and overall prevalence in both genders was $12.5 \%$, higher than the prevalence of successful aging in the United States (11.9\%) and older Malaysians (13.8\%), but far lower than that in Canadian aged 60 and above (42.0\%)[36-38]. For 60-74 years old 
group, we found that the association between successful aging and mortality was in females $(\mathrm{HR}=3.10595 \%, \mathrm{Cl}=1.128-$ 8.543), but was not in males. Gender differences in mortality risk was found across 5 components of successful aging; the association was strongest for presence of major diseases, disability, low cognitive function, and depressive symptoms in females, but for presence of major diseases and disability in males. For those aged 75 and above, the association between successful aging and mortality were not found in both genders. The association was strongest for presence of major diseases in males, but for presence of low cognitive function in females.

Our finding is accordance with previous studies which found successful aging was significantly associated with lower mortality. In a Korean longitudinal study of aging (2006-2014) which included 3848 participants aged 65 and above, nonsuccessful aging older had a higher risk of mortality than successful agers (men: $\mathrm{HR}=1.69,95 \% \mathrm{Cl}=1.18-2.43$; and women: $\mathrm{HR}=2.37,95 \%, \mathrm{Cl}=1.21-4.63)[16]$. Meanwhile, this study found gender differences in mortality risks across all components of successful aging (absence of major illness, freedom from disability, no depressive symptoms, active social engagement, satisfaction with life, high cognitive function, high physical function)[16]. A study(2 year followed survey)from longevity areas of China focusing on the relationship between successful aging index (assessed by self-rated health, depressive symptoms, cognitive function, disability, and physical activities) and the survival status included 2296 old people (65 years old and above). According to this research, the mortality rate in the successful aging group was lower than non-successful aging group, the death rate in successful aging group reduced by $38 \%(\mathrm{HR}=0.62,95 \% \mathrm{Cl}=0.49-0.79)[39]$. Considering the definitions of successful aging varied from different viewpoints, we cannot directly compare those previously available studies with ours. However, from another perspectives, it is proved that achieving successful aging is crucial for lowering rate of mortality and improving life-expectancy, which could support our study.

We can conclude that aging successfully may be beneficial for lower the risk of mortality, but components of successful aging had different contributions to mortality varying from genders. Some studies indicated this gender difference may result from biological, genetic, and social variations[40-43]. Our study showed that the significant association between successful aging and mortality only existed in females (60-74 years old). On the one hand, possible explanations for the phenomenon is that the association between all components of successful aging (except "Active social engagement in life") and mortality was significant in females (60-74 years old), however, there only found significant association between partly components of successful aging ("Major disease" and "Disability") and mortality in males, as a result, the overall effect of successful aging on mortality in males was diminished. On the other hand, possible explanations for the association between the specific components of successful aging ("Depressive symptoms" and "Cognitive function") and mortality may be weaker in males than females might be that females may have greater and more prolonged Hypothalamic-Pituitary-Adrenal (HPA) response to challenge` at older ages than males. That is to say, post-menopausal females are at increased risk of exhibiting greater and more prolonged HPA activation, which may contribute significantly to the increasing risks among such post-menopausal females for chronic diseases, depression, disability and so on[44], previous researches had proved those diseases are associated with higher mortality.

Meanwhile, the association mentioned above diminished with increasing age, that is to say, we cannot observe similar correlation at peopled aged 75 and above among males and females. Potential explanation for the declines in the effects of successful aging on mortality with age is that physiological factors gradually become the prominent contribution factors more than others such as diseases, behaviors and so on for the death of 75 years old and above group.

It is the first longitudinal study using national cohort data to research the educational effects on the association between mortality and successful aging, the study showed that the effect only existed in females aged 65-74 years old group with lower education. On the one hand, focusing on the role of education rather than SES which usually was a comprehensive concept (including education, occupation, income, social class, physical health and so on) provides us a precisely perspective to understand the connection between successful aging and mortality. On the other hand, some studies had found that the effect of education on mortality is stronger in young old than in oldest old[45], lower education population had less possibility to access to more health resources which are related with good health and survival[46, 47]. 
This study had several limitations. First, complete data about causes of death were not available, hindering us from further research of the association between successful aging and cause-specific mortality. Second, misclassification and recall bias might have existed because CHARLS included self-reported data. For instance, recall bias may occur according to memories of the elderly when answering the question about diagnosis of a chronic disease. Third, this study was based on the 4-year follow-up period, further study is needed to consider and research whether education could modify mortality in a longer period of follow-up. Last, our results cannot generalize to a broader range of ages, because of our study design which excluded people aged 45-59 in CHARLS.

\section{Conclusions}

In conclusion, we only found the association between successful aging and mortality in female older aged 60-74 years old, not in males. That is to say, the successful aging female older aged 60-74 years old were more likely to live longevity than those who did not. Moreover, the association between successful aging and its components and mortality existed gender differences, that is to say, physical health is significantly associated with the achieving of successful aging among young older and this study suggested that more measures should be paid on improving mental health among the young female older with lower education to achieve successful aging and to against mortality and live longevity.

\section{List Of Abbreviations}

CHARLS

China Health and Retirement Longitudinal Study; ADL:Activities of Daily Living; W1:The baseline wave; W2:Wave 2; W3:Wave 3; W4:Wave 4 SES:Socioeconomic Status; HRs:Hazard Rations; CLs:Confidence Intervals; TICS:Telephone Interview of Cognitive Status; CES-D:Epidemiological Studies Depression Scale; HPA:Hypothalamic-Pituitary-Adrena.

\section{Declarations}

\section{Ethics approval and consent to participate}

The Biomedical Ethics Review Committee of Peking University (IRB00001052-11015) gave the ethic approval and allowed the CHARLS research group to collect data and all the participants provided signed informed consent at the time of participation. The study methodology was carried out in accordance with approved guidelines.

\section{Consent for publication}

Not applicable

\section{Availability of data and materials}

The dataset collected and analyzed in the current study are available from the corresponding author on reasonable request.

\section{Competing interests}

The authors declare that there are no conflicts of interest.

\section{Funding}

This research did not receive any specific grant from funding agencies in the public, commercial, or not-for-profit sectors.

\section{Author contributions}


Peiya Cao and Huiqiang Luo designed the study, performed the experiments, analyzed the data, and prepared the manuscript, Peiya Cao and Huiqiang Luo were co-first author; Jijie Li analyzed the data; Xiaohui Ren helped perform the analyses, participated in constructive discussions and contributed to the conception of the study.

\section{Acknowledgments}

We are grateful to the China Center for Economic Research at Beijing University for providing us with the data, and we thank the CHARLS research and field team for collecting the data.

\section{References}

1. Christensen K, Doblhammer G, Rau R, Vaupel JW: Ageing populations: the challenges ahead. The Lancet 2009, 374(9696):1196-1208.

2. National Bureau of Statistics. Statistical Communique on National Economic and Social Development [http://www.stats.gov.cn/tjsj/zxfb/201602/t20160229_1323991.html] Accessed 30 Oct 2020.

3. United Nations. World Population Prospects: The 2015 Revision. New York: the United Nations. 2015.

4. Xin C, Zhang B, Fang S, Zhou J: Daytime napping and successful aging among older adults in China: a cross-sectional study. BMC Geriatrics 2020, 20(1):2.

5. Kennedy BK, Berger SL, Brunet A, Campisi J, Cuervo AM, Epel ES, Franceschi C, Lithgow GJ, Morimoto RI, Pessin JE et al: Geroscience: linking aging to chronic disease. Cell 2014, 159(4):709-713.

6. Diehr PH, Thielke SM, Newman AB, Hirsch C, Tracy R: Decline in health for older adults: five-year change in 13 key measures of standardized health. The journals of gerontology Series A, Biological sciences and medical sciences 2013, 68(9):1059-1067.

7. Gutiérrez M, Calatayud P, Tomás J-M: Motives to practice exercise in old age and successful aging: A latent class analysis. Archives of Gerontology and Geriatrics 2018, 77:44-50.

8. Swindell WR, Ensrud KE, Cawthon PM, Cauley JA, Cummings SR, Miller RA, Study Of Osteoporotic Fractures Research G: Indicators of "Healthy Aging" in older women (65-69 years of age). A data-mining approach based on prediction of long-term survival. BMC Geriatrics 2010, 10(1):55.

9. Kusumastuti S, Derks MGM, Tellier S, Di Nucci E, Lund R, Mortensen EL, Westendorp RGJ: Successful ageing: A study of the literature using citation network analysis. Maturitas 2016, 93:4-12.

10. Dillaway HE, Byrnes M: Reconsidering successful aging: A call for renewed and expanded academic critiques and conceptualizations. Journal of Applied Gerontology 2009, 28(6):702-722.

11. Havighurst RJ: Successful Aging. The Gerontologist 1961, 1(1):8-13.

12. Rowe JW, Kahn RL: Successful Aging and Disease Prevention. Advances in Renal Replacement Therapy 2000, 7(1):7077.

13. Lu W, Pikhart H, Sacker A: Domains and Measurements of Healthy Aging in Epidemiological Studies: A Review. Gerontologist 2019, 59(4):E294-E310.

14. Lucas HM, Lozano CJ, Valdez LP, Manzarate R, Lumawag FAJ: A grounded theory of successful aging among select incarcerated older Filipino women. Archives of Gerontology and Geriatrics 2018, 77:96-102.

15. Nosraty L, Pulkki J, Raitanen J, Enroth L, Jylha M: Successful Aging as a Predictor of Long-Term Care Among Oldest Old: The Vitality 90+Study. Journal of Applied Gerontology 2019, 38(4):553-571.

16. Kim H-J, Min J-Y, Min K-B: Successful Aging and Mortality Risk: The Korean Longitudinal Study of Aging (2006-2014). Journal of the American Medical Directors Association 2019, 20(8):1013-1020.

17. Seemungal TA, Hurst JR, Wedzicha JA: Exacerbation rate, health status and mortality in COPD-a review of potential interventions. International journal of chronic obstructive pulmonary disease 2009, 4:203-223. 
18. Wu L-W, Chen W-L, Peng T-C, Chiang S-T, Yang H-F, Sun Y-S, Chan JY-H, Kao T-W: All-cause mortality risk in elderly individuals with disabilities: a retrospective observational study. Bmj Open 2016, 6(9).

19. Schulz R, Beach SR, Ives DG, Martire LM, Ariyo AA, Kop WJ: Association Between Depression and Mortality in Older Adults: The Cardiovascular Health Study. Archives of Internal Medicine 2000, 160(12):1761-1768.

20. Andersen K, Nybo H, Gaist D, Petersen HC, McGue M, Jeune B, Vaupel JW, Christensen K: Cognitive impairment and mortality among nonagenarians: the Danish 1905 cohort survey. Dementia and geriatric cognitive disorders 2002, 13(3):156-163.

21. Gale CR, Martyn CN, Cooper C, Sayer AA: Grip strength, body composition, and mortality. Int J Epidemio/ 2007, 36(1):228-235.

22. Galobardes B, Lynch J, Smith GD: Measuring socioeconomic position in health research. British Medical Bulletin 2007, 81-82:21-37.

23. Cho J: Successful aging and developmental adaptation of oldest-old adults. 2011.

24. Foscolou A, Magriplis E, Tyrovolas S, Chrysohoou C, Sidossis L, Matalas AL, Rallidis L, Panagiotakos D: The association of protein and carbohydrate intake with successful aging: a combined analysis of two epidemiological studies. European journal of nutrition 2019, 58(2):807-817.

25. Berkman LF, Seeman TE, Albert M, Blazer D, Kahn R, Mohs R, Finch C, Schneider E, Cotman C, McClearn G et al: High, usual and impaired functioning in community-dwelling older men and women: findings from the MacArthur Foundation Research Network on Successful Aging. Journal of clinical epidemiology 1993, 46(10):1129-1140.

26. Garfein AJ, Herzog AR: Robust Aging among the Young-Old, Old-Old, and Oldest-Old. The Journals of Gerontology: Series B 1995, 50B(2):S77-S87.

27. Jorm AF, Christensen H, Henderson AS, Jacomb PA, Korten AE, Mackinnon A: Factors Associated with Successful Ageing. Australasian Journal on Ageing 1998, 17(1):33-37.

28. Chou KL, Chi I: Successful aging among the young-old, old-old, and oldest-old Chinese. Int J Aging Hum Dev 2002, 54(1):1-14.

29. Li C, Wu W, Jin H, Zhang X, Xue H, He Y, Xiao S, Jeste DV, Zhang M: Successful aging in Shanghai, China: definition, distribution and related factors. International Psychogeriatrics 2006, 18(3):551-563.

30. Kubzansky LD, Berkman LF, Glass TA, Seeman TE: Is educational attainment associated with shared determinants of health in the elderly? Findings from the MacArthur Studies of Successful Aging. Psychosomatic medicine 1998, 60(5):578-585.

31. Ng TP, Broekman BFP, Niti M, Gwee X, Kua EH: Determinants of Successful Aging Using a Multidimensional Definition Among Chinese Elderly in Singapore. American Journal of Geriatric Psychiatry 2009, 17(5):407-416.

32. Zhao Y, Hu Y, Smith JP, Strauss J, Yang G: Cohort Profile: The China Health and Retirement Longitudinal Study (CHARLS). International Journal of Epidemiology 2014, 43(1):61-68.

33. Pan C-W, Cong X-L, Zhou H-J, Wang X-Z, Sun H-P, Xu Y, Wang P: Evaluating health-related quality of life impact of chronic conditions among older adults from a rural town in Suzhou, China. Archives of Gerontology and Geriatrics 2018, 76:6-11.

34. Katz S, Ford AB, Heiple KG, Newill VA: Studies of Illness in the Aged: Recovery After Fracture of the Hip. Journal of Gerontology 1964, 19(3):285-293.

35. Liu H, Byles JE, Xu X, Zhang M, Wu X, Hall JJ: Association between nighttime sleep and successful aging among older Chinese people. Sleep Medicine 2016, 22:18-24.

36. McLaughlin SJ, Connell CM, Heeringa SG, Li LW, Roberts JS: Successful aging in the United States: prevalence estimates from a national sample of older adults. The journals of gerontology Series B, Psychological sciences and social sciences $2010,65 b(2): 216-226$. 
37. Hamid TA, Momtaz YA, Ibrahim R: Predictors and prevalence of successful aging among older Malaysians. Gerontology 2012, 58(4):366-370.

38. Meng X, D'Arcy C: Successful aging in Canada: prevalence and predictors from a population-based sample of older adults. Gerontology 2014, 60(1):65-72.

39. Shi WH, Lyu YB, Luo JS, Yin ZX, Shi XM: Relationship between the successful aging and survival status among the elderly from longevity areas in China. Zhonghua yu fang yi xue za zhi [Chinese journal of preventive medicine] 2017, 51(11):1024-1027.

40. Back JH, Lee Y: Gender differences in the association between socioeconomic status (SES) and depressive symptoms in older adults. Archives of Gerontology and Geriatrics 2011, 52(3):E140-E144.

41. Brownhill S, Wilhelm K, Barclay L, Schmied V: 'Big build': hidden depression in men. Australian and New Zealand Journal of Psychiatry 2005, 39(10):921-931.

42. Gold CH, Malmberg B, McClearn GE, Pedersen NL, Berg S: Gender and health: A study of older unlike-sex twins. Journals of Gerontology Series B-Psychological Sciences and Social Sciences 2002, 57(3):S168-S176.

43. Wheaton FV, Crimmins EM: Female disability disadvantage: a global perspective on sex differences in physical function and disability. Ageing \& Society 2016, 36(6):1136-1156.

44. Seeman TE, Singer B, Charpentier P: Gender differences in patterns of HPA axis response to challenge: MacArthur studies of successful aging. Psychoneuroendocrinology 1995, 20(7):711-725.

45. Luo Y, Zhang Z, Gu D: Education and mortality among older adults in China. Social Science \& Medicine 2015, 127:134142.

46. Marmot M, Shipley M, Brunner E, Hemingway H: Relative contribution of early life and adult socioeconomic factors to adult morbidity in the Whitehall II study. Journal of Epidemiology and Community Health 2001, 55(5):301-307.

47. Lantz PM, House JS, Lepkowski JM, Williams DR, Mero RP, Chen J: Socioeconomic factors, health behaviors, and mortality: Results from a nationally representative prospective study of US adults. Journal of the American Medical Association 1998, 279(21):1703-1708.

\section{Figures}


Enrolled older $(\geqslant 60$ years older)

$\mathrm{n}=10,517(100 \%)$

Excluded sample with missing or invalid demographic information $(n=1,190)$

1. No education data $(n=1,133)$

2. No marital status data $(\mathrm{n}=2)$

3 . No smoking data $(n=49)$

4. No drinking data $(n=6)$

Excluded sample with two missing key variables

1. No successful aging data $(n=3,784)$

2. No mortality information data $(n=722)$

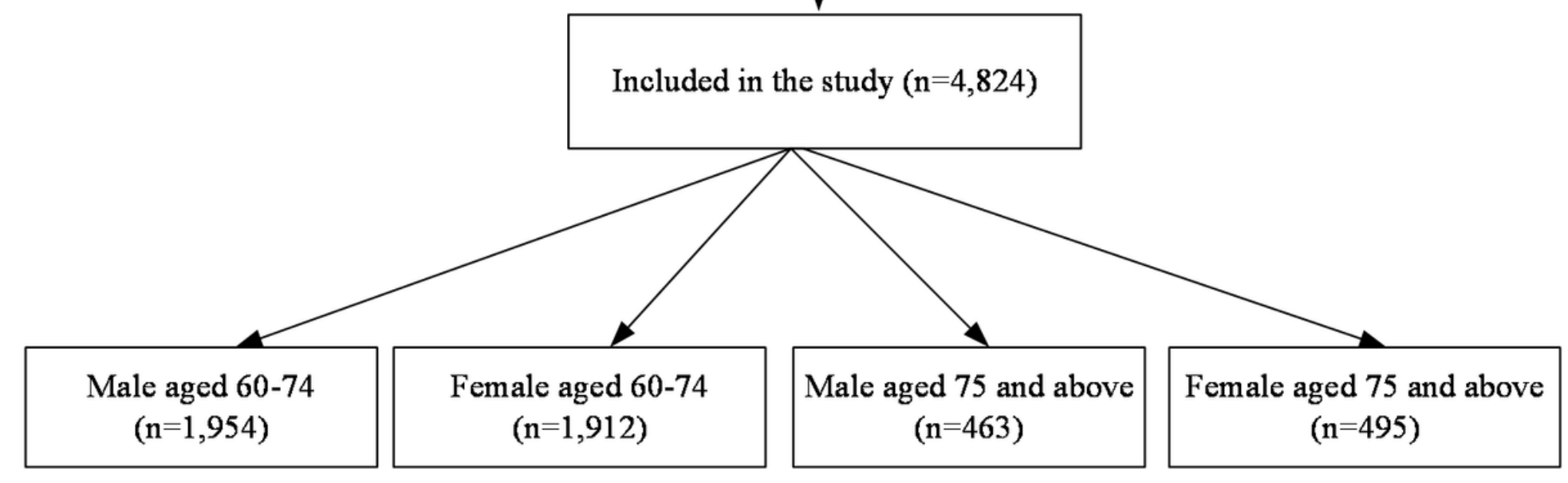

Figure 1

Sample included in the study 